\title{
A One-Vertex Decomposition Algorithm for Generating Algebraic Expressions of Square Rhomboids
}

\author{
Mark Korenblit ${ }^{1}$ and Vadim E. Levit ${ }^{2}$ \\ ${ }^{1}$ Holon Institute of Technology, Israel \\ korenblit@hit.ac.il \\ 2 Ariel University Center of Samaria, Israel \\ levitv@ariel.ac.il
}

\begin{abstract}
The paper investigates relationship between algebraic expressions and graphs. We consider a digraph called a square rhomboid that is an example of non-seriesparallel graphs. Our intention is to simplify the expressions of square rhomboids and eventually find their shortest representations. With that end in view, we describe the new algorithm for generating square rhomboid expressions, which improves on our previous algorithms.
\end{abstract}

\section{Introduction}

A graph $G=(V, E)$ consists of a vertex set $V$ and an edge set $E$, where each edge corresponds to a pair $(v, w)$ of vertices. A graph in which each edge has a weight associated with it is called a network. A graph $G^{\prime}=\left(V^{\prime}, E^{\prime}\right)$ is a subgraph of $G=(V, E)$ if $V^{\prime} \subseteq V$ and $E^{\prime} \subseteq E$. A graph $G$ is a homeomorph of $G^{\prime}$ if $G$ can be obtained by subdividing edges of $G^{\prime}$ with new vertices. We say that a graph $G^{2}=\left(V, E^{\prime}\right)$ is a square of a graph $G=(V, E)$ if $E^{\prime}=\{(u, w):(u, w) \in E \vee((u, v) \in E \wedge(v, w) \in E)$ for some $v \in V\}$. A two-terminal directed acyclic graph $(s t-d a g)$ has only one source and only one sink.

We consider a labeled graph which has labels attached to its edges. Each path between the source and the sink (a sequential path) in an st-dag can be presented by a product of all edge labels of the path. We define the sum of edge label products corresponding to all possible sequential paths of an st-dag $G$ as the canonical expression of $G$. An algebraic expression is called an st-dag expression (a factoring of an st-dag in [4]) if it is algebraically equivalent to the canonical expression of an st-dag. An st-dag expression consists of literals (edge labels), and the operators + (disjoint union) and . 


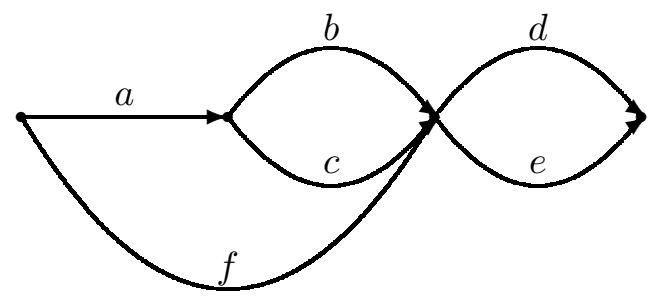

Figure 1.1: A series-parallel graph.

(concatenation, also denoted by juxtaposition). An expression of an st-dag $G$ will be hereafter denoted by $\operatorname{Ex}(G)$.

We define the total number of literals in an algebraic expression as the complexity of the algebraic expression. An equivalent expression with the minimum complexity is called an optimal representation of the algebraic expression.

A series-parallel graph is defined recursively so that a single edge is a series-parallel graph and a graph obtained by a parallel or a series composition of series-parallel graphs is series-parallel. As shown in [4] and [12], a series-parallel graph expression has a representation in which each literal appears only once. This representation is an optimal representation of the series-parallel graph expression. For example, the canonical expression of the series-parallel graph presented in Figure 1.1 is $a b d+a b e+a c d+a c e+f e+f d$. Since it is a series-parallel graph, the expression can be reduced to $(a(b+c)+f)(d+e)$, where each literal appears once.

A Fibonacci graph [10] has vertices $\{1,2,3, \ldots, n\}$ and edges $\{(v, v+1) \mid v=1,2, \ldots$, $n-1\} \cup\{(v, v+2) \mid v=1,2, \ldots, n-2\}$. As shown in [5], an st-dag is series-parallel if and only if it does not contain a subgraph which is a homeomorph of the forbidden subgraph positioned between vertices 1 and 4 of the Fibonacci graph illustrated in Figure 1.2. Thus, a Fibonacci graph gives a generic example of non-series-parallel graphs.

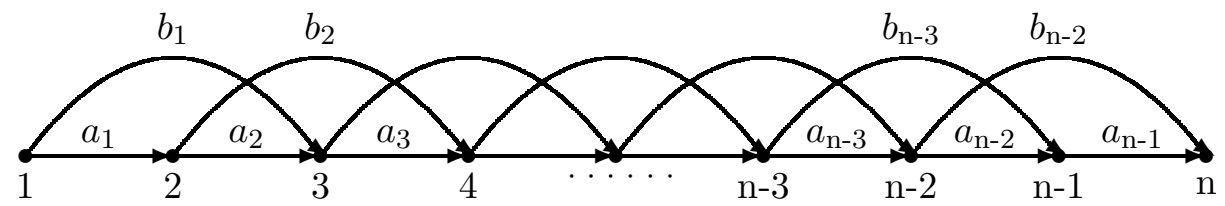

Figure 1.2: A Fibonacci graph.

Mutual relations between graphs and expressions are discussed in a number of works. Specifically, [18], [19], and [25] consider the correspondence between seriesparallel graphs and read-once functions. A Boolean function is defined as read-once if it may be computed by some formula in which no variable occurs more than once (readonce formula). On the other hand, a series-parallel graph expression can be reduced to the representation in which each literal appears only once. Hence, such a representation 
of a series-parallel graph expression can be considered as a read-once formula (boolean operations are replaced by arithmetic ones).

Problems related to computations on graphs have applications in different areas.

Specifically, many network problems, which are either intractable or have complicated solutions in the general case are solvable for series-parallel graphs. For example, some efficient algorithms for flow problems on series-parallel networks are presented in [2], [3], [11], [26]. Papers [1], [6], [16], [17] consider sequencing and scheduling in relation to precedence series-parallel constraints. Linear algorithms for reliability problems on series-parallel networks are presented in [24], [27].

An expression of a homeomorph of the forbidden subgraph belonging to any nonseries-parallel st-dag has no representation in which each literal appears once. For example, consider the subgraph positioned between vertices 1 and 4 of the Fibonacci graph shown in Figure 1.2. Possible optimal representations of its expression are $a_{1}\left(a_{2} a_{3}+b_{2}\right)+b_{1} a_{3}$ or $\left(a_{1} a_{2}+b_{1}\right) a_{3}+a_{1} b_{2}$. For this reason, an expression of a nonseries-parallel st-dag can not be represented as a read-once formula. However, for arbitrary functions, which are not read-once, generating the optimum factored form is NP-complete [28].

The problem of factoring boolean functions into shorter, more compact formulae is one of the basic operations in algorithmic logic synthesis. In logic synthesis, one standard measure of the complexity of a logic circuit is the number of literals. Computation time also depends on the number of literals. Some algorithms developed in order to obtain good factored forms are described in [8], [9].

A symbolic approach to scheduling of a robotic line is considered in [15]. The method uses the max-algebra tools and allows the shortest-path problem to be interpreted as the computation of the st-dag expression. The complexity of this problem is determined by the complexity of the st-dag expression. For a robotic line represented by a Fibonacci graph, the proposed algorithm generates the processing sequence in polynomial time.

A method for automated composition of algebraic expressions in complex business process modeling based on acyclic directed graph reductions is introduced in [20]. The method transforms business step dependencies described by users into digraphs and finally generates algebraic expressions. If a graph is not series-parallel, the algorithm checks potential structural conflicts whose presence complicates certain aspects, such as execution control and system scalability. In this case, the expression generation may require exponential time.

A lot of problems have polynomial algorithms on planar graphs, i.e., the graphs that can be drawn in the plane without any edges crossing. For example, some polynomialtime algorithms for the exact computation of network reliability applied to planar graphs are surveyed in [21]. In [22] a polynomial algorithm for the connectivity augmentation problem is presented. This problem has applications in upgrading telecommunication networks to be invulnerable to link or node failures. There are also many practical 
applications with a graph structure in which crossing edges are a nuisance, including design problems for circuits, subways, utility lines [7].

In our work we consider expressions with a minimum (or, at least, a polynomial) complexity as a key to generating efficient algorithms on distributed systems.

In [12] we presented an algorithm, which generates the expression of $O\left(n^{2}\right)$ complexity for an $n$-vertex Fibonacci graph.

In this paper we investigate a non-series-parallel st-dag called a square rhomboid (Figure 1.3). This graph looks like a planar approximation of the square of a rhomboid, which is a series composition of rhomb graphs. A square rhomboid consists of the same vertices as the corresponding rhomboid. However, edges labeled by letters $a, b$, and $c$ (see Figure 1.3) are absent in a rhomboid. Geometrically, a square rhomboid can be considered to be a "gluing" of two Fibonacci graphs, i.e., it is the next harder one in a sequence of increasingly non-series-parallel graphs.

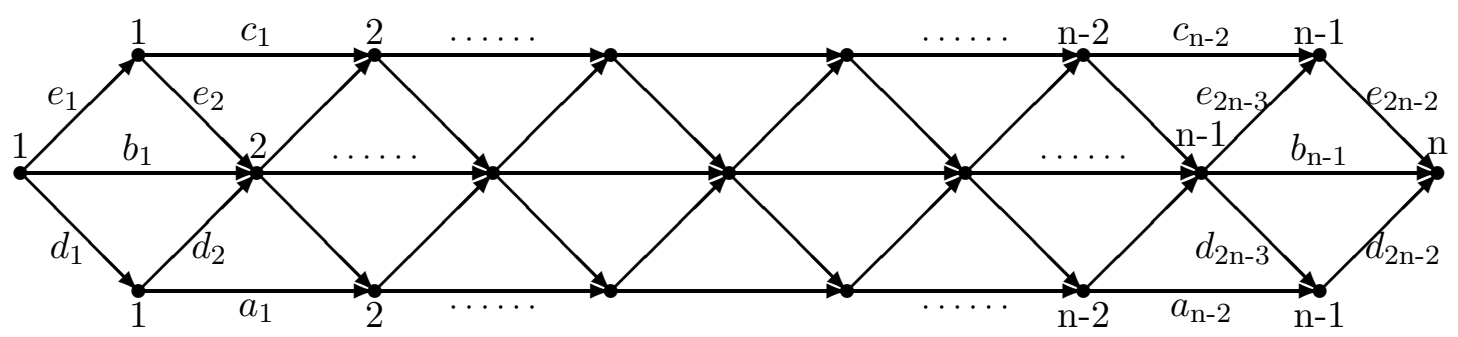

Figure 1.3: A square rhomboid of size $n$.

The set of vertices of an $N$-vertex square rhomboid consists of $\frac{N+2}{3}$ middle (basic), $\frac{N-1}{3}$ upper, and $\frac{N-1}{3}$ lower vertices. Upper and lower vertices numbered $x$ will be denoted in formulae by $\bar{x}$ and $\underline{x}$, respectively. The square rhomboid ( $S R$ for brevity) including $n$ basic vertices will be denoted by $S R(n)$ and will be called an $S R$ of size $n$.

Our intention in this paper is to simplify the expressions of square rhomboids and eventually find their optimal representations. With that end in view, we present an algorithm based on a decomposition method.

\section{A One-Vertex Decomposition Method (1-VDM)}

The method is based on revealing subgraphs in the initial graph. The resulting expression is produced by a special composition of subexpressions describing these subgraphs.

For a non-trivial $S R$ subgraph with a source $p$ and a sink $q$ we choose any decomposition vertex $i(p<i<q)$ located in the basic group of a subgraph. We conditionally split each $S R$ through its decomposition vertex (see the example in Figure 2.1). 


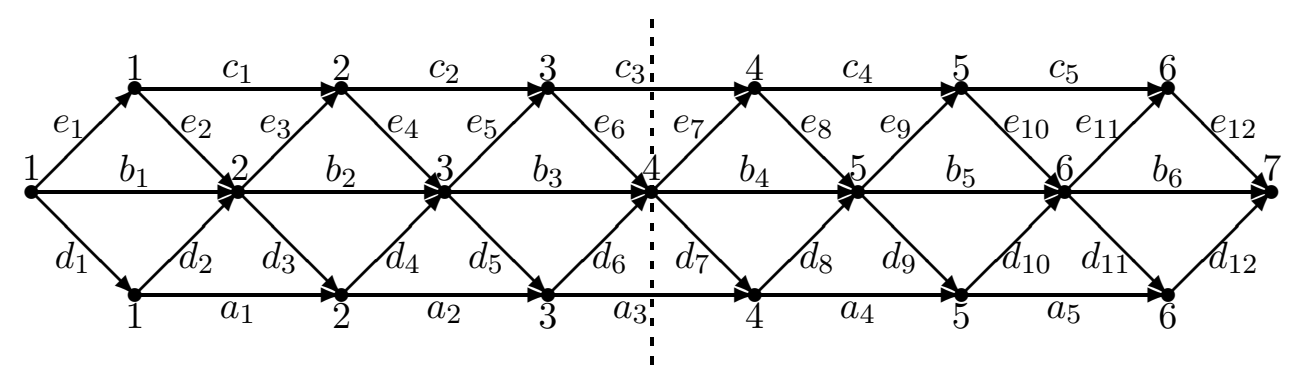

Figure 2.1: Decomposition of a square rhomboid of size 7 at vertex 4 .

Two kinds of subgraphs are revealed in the graph in the course of decomposition. The first of them is an $S R$ with a fewer number of vertices than the initial $S R$. The second one is an $S R$ supplemented by two additional edges at one of four sides. Possible varieties of this st-dag (we call it a single-leaf square rhomboid and denote by $\widehat{S R}$ ) which are subgraphs revealed from an $S R$ in Figure 2.1, are illustrated in Figure 2.2(a, b, c, d). Let $\widehat{S R}(n)$ (an $\widehat{S R}$ of size $n$ ) denote an $\widehat{S R}$ including $n$ basic vertices.

We denote by $E(p, q)$ a subexpression related to an $S R$ subgraph with a source $p$ and a sink $q$. We denote by $E(p, \bar{q}), E(\bar{p}, q), E(p, \underline{q}), E(\underline{p}, q)$ subexpressions related to $\widehat{S R}$ subgraphs of Figure 2.2(a, b, c, d, respectively) with a source $p$ and a sink $\bar{q}$, a source $\bar{p}$ and a sink $q$, a source $p$ and a sink $\underline{q}$, and a source $\underline{p}$ and a sink $q$.

(a)

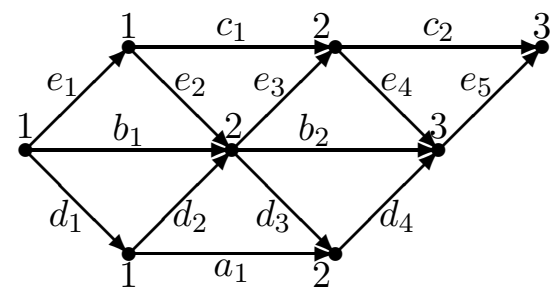

(c)

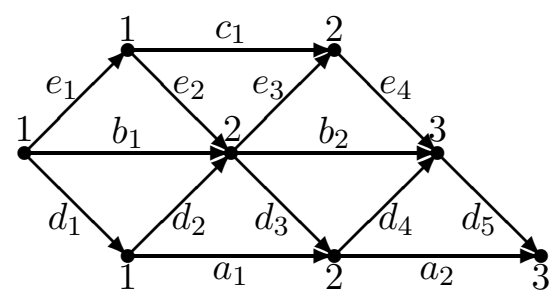

(b)

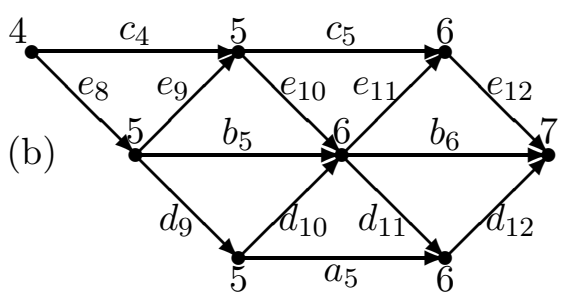

(d)

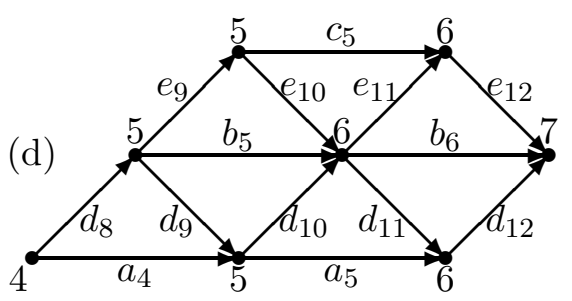

Figure 2.2: Varieties of a single-leaf square rhomboid of size 3.

Any path from vertex 1 to vertex 7 in Fig. 2.1 passes through decomposition vertex 4 or through edge $c_{3}$ or through edge $a_{3}$. Therefore, $E(p, q)$ is generated by the following recursive procedure (decomposition procedure): 
1. case $q=p: E(p, q) \leftarrow 1$

2. case $q=p+1: E(p, q) \leftarrow b_{p}+e_{2 p-1} e_{2 p}+d_{2 p-1} d_{2 p}$

3. case $q>p+1: \operatorname{choice}(p, q, i)$

4 .

$$
\begin{aligned}
E(p, q) \leftarrow & E(p, i) E(i, q)+E(p, \overline{i-1}) c_{i-1} E(\bar{i}, q)+ \\
& E(p, \underline{i-1}) a_{i-1} E(\underline{i}, q)
\end{aligned}
$$

The expression related to a one-vertex $S R$ is defined formally as 1 (line 1$)$. Line 2 describes the expression of $S R(2)$ which is a trivial subgraph. The procedure choice $(p, q, i)$ in line 3 chooses an arbitrary number $i$ on the interval $(p, q)$ so that $p<i<q$. This number is a number of a decomposition vertex in a basic group of the $S R$ subgraph positioned between vertices $p$ and $q$. A current subgraph is decomposed into six new subgraphs in line 4 . Subgraphs described by subexpressions $E(p, i)$ and $E(i, q)$ include all paths from vertex $p$ to vertex $q$ passing through vertex $i$. Subgraphs described by subexpressions $E(p, \overline{i-1})$ and $E(\bar{i}, q)$ include all paths from vertex $p$ to vertex $q$ passing via edge $c_{i-1}$. Subgraphs described by subexpressions $E(p, \underline{i-1})$ and $E(\underline{i}, q)$ include all paths from vertex $p$ to vertex $q$ passing via edge $a_{i-1}$.

$E(1, n)$ is the expression of the initial $S R$ of size $n$. Hence, the decomposition procedure is initially invoked by substituting 1 and $n$ instead of $p$ and $q$, respectively.

We now consider the algorithm that generates the algebraic expression for a square rhomboid using 1-VDM as its base.

\section{A One-Vertex Decomposition Algorithm (1-VDA)}

The algorithm is based on decomposition of the initial graph and all kinds of subgraphs to new subgraphs.

We conjecture that the representation with the minimum complexity of $\operatorname{Ex}(S R)$ derived by $1-\mathrm{VDM}$ is generated when the decomposition vertex is a middle vertex in the basic group of the $S R$. That is, the number $i$ of the decomposition vertex for a current $S R$ subgraph which is positioned between vertices $p$ and $q$ is chosen as $\frac{q+p}{2}\left(\left\lceil\frac{q+p}{2}\right\rceil\right.$ or $\left.\left\lfloor\frac{q+p}{2}\right\rfloor\right)$. In other words, $i$ is equal to $\frac{q+p}{2}$ for odd $q-p+1$ and $i$ equals $\frac{q+p-1}{2}$ or $\frac{q+p+1}{2}$ for even $q-p+1$.

An $\widehat{S R}$ subgraph is decomposed through a decomposition vertex selected in its basic group into six new subgraphs in the same way as an $S R$ (see the examples in Figure 3.1). The decomposition vertex is chosen so that the location of the split is in the middle of the subgraph.

Three kinds of subgraphs are revealed in an $\widehat{S R}$ in the course of decomposition. The first and the second of them are an $S R$ and an $\widehat{S R}$, respectively. The third one is an $S R$ supplemented by two additional pairs of edges (one pair is on the left and another one 

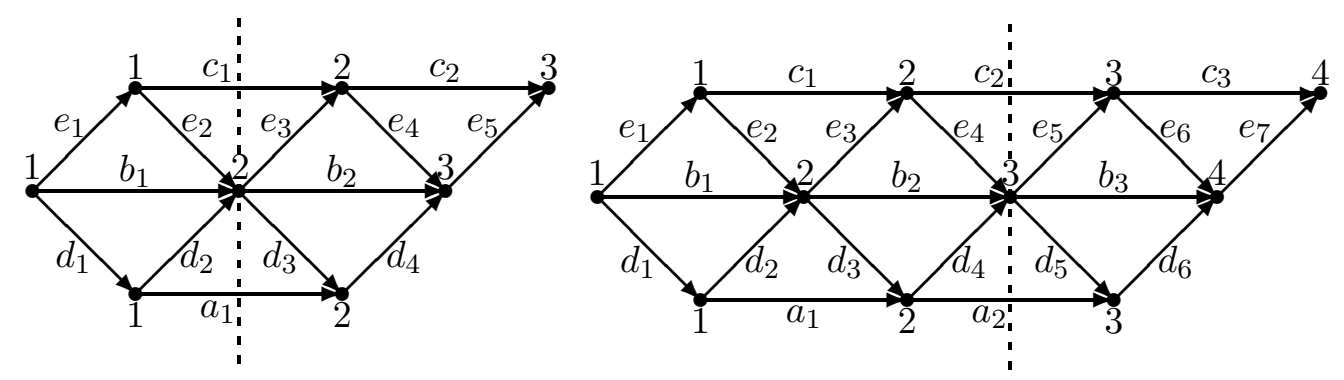

Figure 3.1: Decomposition of single-leaf square rhomboids by 1-VDA.

is on the right). Possible varieties of this st-dag (we call it a dipterous square rhomboid and denote it by $\widehat{\widehat{S R}}$ ) are illustrated in Figure $3.2(\mathrm{a}, \mathrm{b}, \mathrm{c}, \mathrm{d})$. We define subgraphs illustrated in Figure $3.2(\mathrm{a}, \mathrm{c})$ as trapezoidal $\widehat{\widehat{S R}}$ graphs and subgraphs illustrated in Figure $3.2(\mathrm{~b}, \mathrm{~d})$ as parallelogram $\widehat{\widehat{S R}}$ graphs. Let $\widehat{\widehat{S R}}(n)$ (an $\widehat{\widehat{S R}}$ of size $n$ ) denote an $\widehat{\widehat{S R}}$ including $n$ basic vertices.

We denote by $E(\bar{p}, \bar{q}), E(\underline{p}, \bar{q}), E(\underline{p}, \underline{q}), E(\bar{p}, \underline{q})$ subexpressions related to subgraphs of Figure 3.2(a, b, c, d, respectively) with a source $\bar{p}$ and a sink $\bar{q}$, a source $\underline{p}$ and a sink $\bar{q}$, a source $\underline{p}$ and a sink $\underline{q}$, and a source $\bar{p}$ and a sink $\underline{q}$.

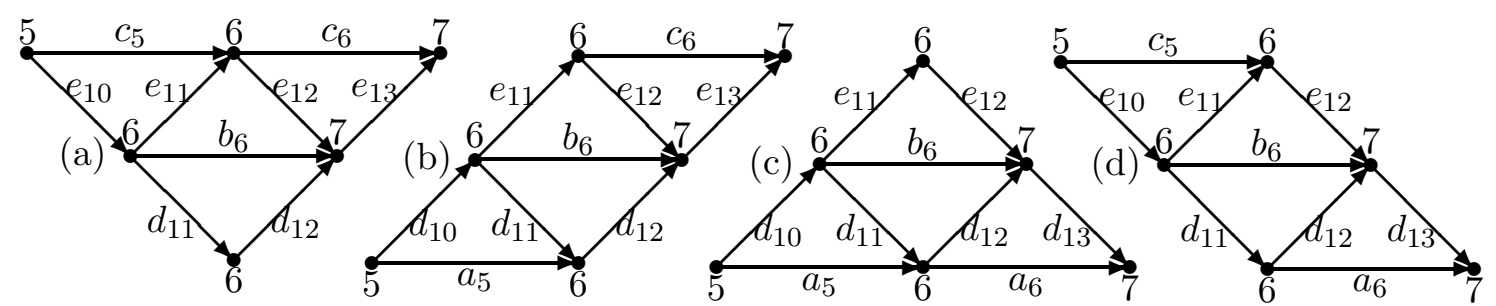

Figure 3.2: Varieties of a dipterous square rhomboid of size 2 .

An $\widehat{\widehat{S R}}$ subgraph is decomposed into six new subgraphs in the same way as an $S R$ and an $\widehat{S R}$ (see the examples in Figure 3.3). The number $i$ of the decomposition vertex in the basic group for a current $\widehat{\widehat{S R}}$ subgraph which is positioned between vertices $p$ and $q$, is chosen as $\frac{q+p+1}{2}\left(\left\lceil\frac{q+p+1}{2}\right\rceil\right.$ or $\left.\left\lfloor\frac{q+p+1}{2}\right\rfloor\right)$.

In the course of decomposition, two kinds of subgraphs are revealed in an $\widehat{\widehat{S R}}$. They are an $\widehat{S R}$ and an $\widehat{\widehat{S R}}$. Therefore, no new kinds of subgraphs are formed and subexpressions related to all subgraphs can be generated.

Hence, $E x(S R)$ is computed by the following recursive relations: 


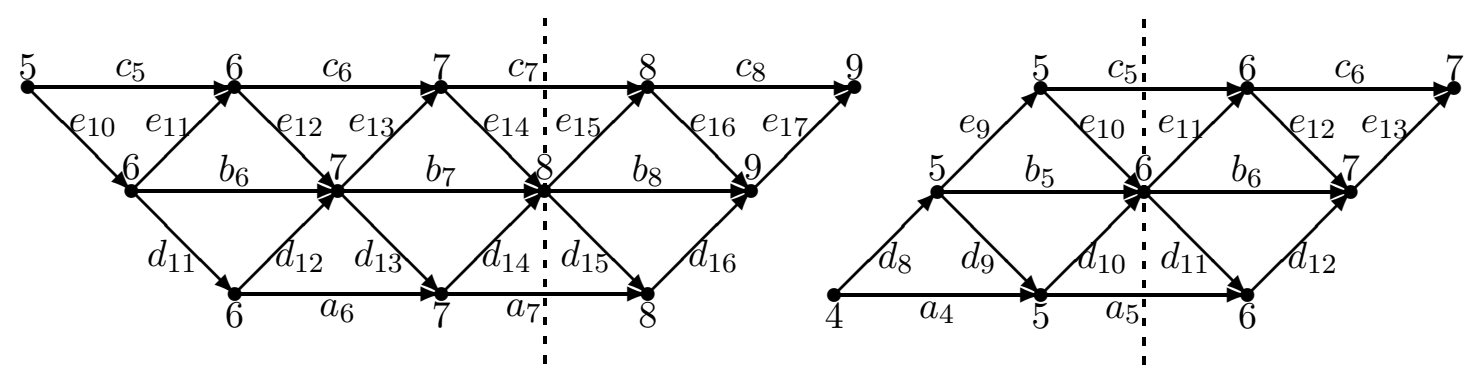

Figure 3.3: Decomposition of dipterous square rhomboids by 1-VDA.

1. $E(p, p)=1$

2. $E(p, \bar{p})=e_{2 p-1} \quad$ 3. $E(p, \underline{p})=d_{2 p-1} \quad$ 4. $E(\bar{p}, p+1)=e_{2 p} \quad$ 5. $E(\underline{p}, p+1)=d_{2 p}$

6. $E(\bar{p}, \overline{p+1})=c_{p}+e_{2 p} e_{2 p+1} \quad$ 7. $E(\bar{p}, p+1)=e_{2 p} d_{2 p+1}$

8. $E(\underline{p}, \overline{p+1})=d_{2 p} e_{2 p+1} \quad$ 9. $E(\underline{p}, \underline{p+1})=a_{p}+d_{2 p} d_{2 p+1}$

10. $E(p, p+1)=b_{p}+e_{2 p-1} e_{2 p}+\bar{d}_{2 p-1} \overline{d_{2 p}}$

11. $E(p, \overline{p+1})=\left(b_{p}+d_{2 p-1} d_{2 p}\right) e_{2 p+1}+e_{2 p-1}\left(c_{p}+e_{2 p} e_{2 p+1}\right)$

12. $E(p, \underline{p+1})=\left(b_{p}+e_{2 p-1} e_{2 p}\right) d_{2 p+1}+d_{2 p-1}\left(a_{p}+d_{2 p} d_{2 p+1}\right)$

13. $E(\bar{p}, \overline{p+2})=\left(c_{p}+e_{2 p} e_{2 p+1}\right) e_{2 p+2}+e_{2 p}\left(b_{p+1}+d_{2 p+1} d_{2 p+2}\right)$

14. $E(p, p+2)=\left(a_{p}+d_{2 p} d_{2 p+1}\right) d_{2 p+2}+d_{2 p}\left(b_{p+1}+e_{2 p+1} e_{2 p+2}\right)$

15. $E(\overline{\bar{p}}, \overline{p+2})=e_{2 p}\left(b_{p+1}+d_{2 p+1} d_{2 p+2}\right) e_{2 p+3}+\left(a_{p}+d_{2 p} d_{2 p+1}\right)\left(a_{p+1}+d_{2 p+2} d_{2 p+3}\right)$

16. $E(\bar{p}, p+2)=e_{2 p}\left(b_{p+1} d_{2 p+3}+d_{2 p+1}\left(a_{p+1}+d_{2 p+2} d_{2 p+3}\right)\right)+\left(c_{p}+e_{2 p} e_{2 p+1}\right) e_{2 p+2} d_{2 p+3}$

17. $E(\underline{p}, \overline{\overline{p+2}})=d_{2 p}\left(b_{p+1} e_{2 p+3}+e_{2 p+1}\left(c_{p+1}+e_{2 p+2} e_{2 p+3}\right)\right)+\left(a_{p}+d_{2 p} d_{2 p+1}\right) d_{2 p+2} e_{2 p+3}$

18. $E(\underline{p}, \underline{p+2})=d_{2 p}\left(b_{p+1}+e_{2 p+1} e_{2 p+2}\right) d_{2 p+3}+\left(c_{p}+e_{2 p} e_{2 p+1}\right)\left(c_{p+1}+e_{2 p+2} e_{2 p+3}\right)$

19. $E(\bar{p}, \bar{q})=E(p, i) E(i, \bar{q})+E(p, \overline{i-1}) c_{i-1} E(\bar{i}, \bar{q})+E(p, \underline{i-1}) a_{i-1} E(\underline{i}, \bar{q}), i=\left\lceil\frac{q+p}{2}\right\rceil$ $(q>p+1)$

20. $E(p, \underline{q})=E(p, i) E(i, \underline{q})+E(p, \overline{i-1}) c_{i-1} E(\bar{i}, \underline{q})+E(p, \underline{i-1}) a_{i-1} E(\underline{i}, \underline{q}), i=\left\lceil\frac{q+p}{2}\right\rceil$ $(q>\bar{p}+1)$

21. $E(\bar{p}, q)=E(\bar{p}, i) E(i, q)+E(\bar{p}, \overline{i-1}) c_{i-1} E(\bar{i}, q)+E(\bar{p}, \underline{i-1}) a_{i-1} E(\underline{i}, q), i=\left\lceil\frac{q+p}{2}\right\rceil$ $(q>p+2)$

22. $E(\underline{p}, q)=E(\underline{p}, i) E(i, q)+E(\underline{p}, \overline{i-1}) c_{i-1} E(\bar{i}, q)+E(\underline{p}, \underline{i-1}) a_{i-1} E(\underline{i}, q), i=\left\lceil\frac{q+p}{2}\right\rceil$ $(q>p+2)$

23. $E(\bar{p}, \bar{q})=E(\bar{p}, i) E(i, \bar{q})+E(\bar{p}, \overline{i-1}) c_{i-1} E(\bar{i}, \bar{q})+E(\bar{p}, \underline{i-1}) a_{i-1} E(\underline{i}, \bar{q}), i=\frac{q+p+1}{2}$ $(q>p+2)$

24. $E(\bar{p}, \underline{q})=E(\bar{p}, i) E(i, \underline{q})+E(\bar{p}, \overline{i-1}) c_{i-1} E(\bar{i}, \underline{q})+E(\bar{p}, \underline{i-1}) a_{i-1} E(\underline{i}, \underline{q}), i=\frac{q+p+1}{2}$ $(q>\bar{p}+2)$

25. $E(\underline{p}, \bar{q})=E(\underline{p}, i) E(i, \bar{q})+E(\underline{p}, \overline{i-1}) c_{i-1} E(\bar{i}, \bar{q})+E(\underline{p}, \underline{i-1}) a_{i-1} E(\underline{i}, \bar{q}), i=\frac{q+p+1}{2}$ $(q>p+2)$

26. $E(\underline{p}, \underline{q})=E(\underline{p}, i) E(i, \underline{q})+E(\underline{p}, \overline{i-1}) c_{i-1} E(\bar{i}, \underline{q})+E(\underline{p}, \underline{i-1}) a_{i-1} E(\underline{i}, \underline{q}), i=\frac{q+p+1}{2}$ $(q>\bar{p}+2)$

27. $E(p, q)=E(p, i) E(i, q)+E(p, \overline{i-1}) c_{i-1} E(\bar{i}, q)+E(p, \underline{i-1}) a_{i-1} E(\underline{i}, q), i=\frac{q+p}{2}$ $(q>p+1)$. 
Subgraphs of sizes 1 and 2 are trivial and their expressions are in lines $(1-18)$. Specifically, expressions of subgraphs $\widehat{S R}(2)$ and $\widehat{\widehat{S R}}(2)$ are presented in the minimum factored form (lines $11-18$ ).

For example, the expression of the square rhomboid of size 3 derived by 1-VDA is

$$
\left(b_{1}+e_{1} e_{2}+d_{1} d_{2}\right)\left(b_{2}+e_{3} e_{4}+d_{3} d_{4}\right)+e_{1} c_{1} e_{4}+d_{1} a_{1} d_{4} .
$$

It contains 16 literals.

The following lemma results from lines $(23-26)$ of 1 -VDA.

Lemma 3.1. Complexities of expressions $\quad$ Ex $($ trapezoidal $\widehat{\widehat{S R}}(n)) \quad$ and Ex (parallelogram $\widehat{\widehat{S R}}(n))$ derived by $1-V D A$ are equal for $n>2$.

The following proposition results from Lemma 3.1 and from lines (1 - 27) of 1-VDA.

Proposition 3.2. The total number of literals $T(n)$ in the expression $\operatorname{Ex}(S R(n))$ derived by 1-VDA is defined recursively as follows:

1) $T(1)=0$;2) $\widehat{T}(1)=1$; 3) $\widehat{\widehat{T}}_{p r}(1)=2$; 4) $\widehat{\widehat{T}}_{t r}(1)=3$

5) $T(2)=5$; 6) $\widehat{T}(2)=8$; 7) $\widehat{\widehat{T}}_{p r}(2)=12$;8) $\widehat{\widehat{T}}_{t r}(2)=11$

9) $\widehat{T}(3)=22$; 10) $\widehat{T}(3)=28$; 11) $\widehat{T}(4)=47$; 12) $\widehat{T}(4)=60$

13) $\widehat{T}(5)=79$; 14) $\widehat{\hat{T}}(5)=92$; 15) $\widehat{T}(6)=132$; 16) $\widehat{\hat{T}}(6)=50$

17) $T(n)=T\left(\left\lceil\frac{n}{2}\right\rceil\right)+T\left(\left\lfloor\frac{n}{2}\right\rfloor+1\right)+2 \widehat{T}\left(\left\lceil\frac{n}{2}\right\rceil-1\right)+2 \widehat{T}\left(\left\lfloor\frac{n}{2}\right\rfloor\right)+2 \quad(n>2)$

18) $\widehat{T}(n)=T\left(\left\lfloor\frac{n}{2}\right\rfloor+1\right)+\widehat{T}\left(\left\lceil\frac{n}{2}\right\rceil\right)+2 \widehat{T}\left(\left\lfloor\frac{n}{2}\right\rfloor\right)+2 \widehat{T}\left(\left\lceil\frac{n}{2}\right\rceil-1\right)+2 \quad(n>6)$

19) $\widehat{\widehat{T}}(n)=\widehat{T}\left(\left\lceil\frac{n}{2}\right\rceil\right)+\widehat{T}\left(\left\lfloor\frac{n}{2}\right\rfloor+1\right)+2 \widehat{\widehat{T}}\left(\left\lceil\frac{n}{2}\right\rceil-1\right)+2 \widehat{\widehat{T}}\left(\left\lfloor\frac{n}{2}\right\rfloor\right)+2 \quad(n>6)$,

where $\widehat{T}(n), \widehat{\widehat{T}}_{p r}(1), \widehat{\widehat{T}}_{t r}(1)$, and $\widehat{\widehat{T}}(n)$ are the total numbers of literals in $\operatorname{Ex}(\widehat{S R}(n))$, Ex $($ parallelogram $\widehat{\widehat{S R}}(1))$, Ex $($ trapezoidal $\widehat{\widehat{S R}}(1))$, and $\operatorname{Ex}(\widehat{\widehat{S R}}(n))$, respectively.

Hence, the expression $\operatorname{Ex}(S R(n))$ derived by 1-VDA consists of six subexpressions related to six revealed subgraphs of size $n^{\prime} \approx \frac{n}{2}$ and two additional literals. Each of these subexpressions is constructed in its turn from six subexpressions related to six subgraphs of size $n^{\prime \prime} \approx \frac{n}{4}$ and two additional literals, etc. Thus, by the master theorem, $T(n)=O\left(n^{\log _{2} 6}\right)$, i.e., 1-VDA provides the representation of $\operatorname{Ex}(S R(n))$ with a polynomial complexity.

It is of interest to obtain exact formulae describing complexity of the expression $\operatorname{Ex}(S R(n))$ derived by 1-VDA. We attempt to do it for $n$ that is a power of two, i.e., 
$n=2^{k}$ for some positive integer $k \geq 2$. Statements $(17-19)$ of Proposition 3.2 are presented for $n=2^{k}(k \geq 3)$ as

$$
\left\{\begin{array}{l}
T(n)=T\left(\frac{n}{2}+1\right)+T\left(\frac{n}{2}\right)+2 \widehat{T}\left(\frac{n}{2}\right)+2 \widehat{T}\left(\frac{n}{2}-1\right)+2 \\
\widehat{T}(n)=T\left(\frac{n}{2}+1\right)+3 \widehat{T}\left(\frac{n}{2}\right)+2 \widehat{\widehat{T}}\left(\frac{n}{2}-1\right)+2 \\
\widehat{\widehat{T}}(n)=\widehat{T}\left(\frac{n}{2}+1\right)+\widehat{T}\left(\frac{n}{2}\right)+2 \widehat{\widehat{T}}\left(\frac{n}{2}\right)+2 \widehat{T}\left(\frac{n}{2}-1\right)+2,
\end{array}\right.
$$

respectively.

After a number of transformations, the following explicit formulae for simultaneous recurrences (3.1) are obtained by the method for linear recurrence relations solving [23]:

$$
\begin{aligned}
& T(n)=\frac{154}{135} n^{\log _{2} 6}+\frac{1}{27} n^{\log _{2} 3}-\frac{2}{5} \\
& \widehat{T}(n)=\frac{154}{135} n^{\log _{2} 6}+\frac{19}{27} n^{\log _{2} 3}-\frac{2}{5} \\
& \widehat{T}(n)=\frac{154}{135} n^{\log _{2} 6}+\frac{58}{27} n^{\log _{2} 3}-\frac{2}{5} .
\end{aligned}
$$

\section{Comparison of 1-VDA with Other Algorithms}

Another decomposition method (called 2-VDM) for generating algebraic expressions of square rhomboids is presented in [13] and [14]. The method consists in splitting a square rhomboid through two decomposition vertices one of which belongs to the upper group and another one to the lower group. The following algorithms based on this method are considered in [13] and [14]: full decomposition algorithm (FDA), combined decomposition algorithm (CDA) and improved FDA (IFDA). Complexities of generated by these algorithms expressions are also $O\left(n^{\log _{2} 6}\right)$. The values of $T(n)$ for these algorithms and for 1 -VDA are presented in Table $4.1(4 \leq n \leq 10)$.

\begin{tabular}{||c||c|c|c|c||}
\hline \hline$n$ & $T(n)$, FDA & $T(n)$, CDA & $T(n)$, IFDA & $T(n), 1$-VDA \\
\hline 4 & 47 & 43 & 43 & 41 \\
\hline 5 & 110 & 102 & 100 & 66 \\
\hline 6 & 173 & 161 & 157 & 119 \\
\hline 7 & 252 & 236 & 228 & 172 \\
\hline 8 & 331 & 311 & 299 & 247 \\
\hline 9 & 520 & 488 & 470 & 322 \\
\hline 10 & 709 & 665 & 641 & 439 \\
\hline \hline
\end{tabular}

Table 4.1: Complexities for FDA, CDA, IFDA, and 1-VDA.

One can see that 1-VDA is more efficient than FDA, CDA, and IFDA. 
The numerical advantage of 1-VDA in contrast to other algorithms follows also from presented in [13] and [14] explicit formulae analogous to (3.2). The coefficient of the leading term of the formulae $-n^{\log _{2} 6}$ is equal to $\frac{79}{45} \approx 1.76$ for FDA, $\frac{227}{135} \approx 1.68$ for CDA, $\frac{212}{135} \approx 1.57$ for IFDA, and $\frac{154}{135} \approx 1.14$ for 1 -VDA.

\section{Conclusions}

The decomposition method divides all subgraphs, which are revealed in the current recursive step into a constant number of subgraphs of proportionally decreasing sizes. The existence of a decomposition method for an st-dag $G$ is a sufficient condition for the existence of a representation of $\operatorname{Ex}(G)$ with a polynomial complexity. The complexity depends on the number of revealed subgraphs in each recursive step.

\section{References}

[1] H. M. Abdel-Wahab and T. Kameda, Scheduling to Minimize Maximum Cumulative Cost Subject to Series-Parallel Precedence Constraints, Operations Research, Vol. 26, No 1, 1978, 141-158.

[2] W. W. Bein and P. Brucker, Greedy Concepts for Network Flow Problems, Discrete Applied Mathematics 10, 1985, 117-124.

[3] W. W. Bein, P. Brucker, and A. Tamir, Minimum Cost Flow Algorithms for SeriesParallel Networks, Discrete Applied Mathematics 15, 1986, 135-144.

[4] W. W. Bein, J. Kamburowski, and M. F. M. Stallmann, Optimal Reduction of Two-Terminal Directed Acyclic Graphs, SIAM Journal of Computing, Vol. 21, No 6, 1992, 1112-1129.

[5] R. J. Duffin, Topology of Series-Parallel Networks, Journal of Mathematical Analysis and Applications 10, 1965, 303-318.

[6] L. Finta, Z. Liu, I. Milis, and E. Bampis, Scheduling UET-UCT Series-Parallel Graphs on Two Processors, Theoretical Computer Science, Vol. 162, No 2, 1996, 323-340.

[7] M. M. Fleck, Planar Graphs, http://www.cs.illinois.edu/class/sp11/cs173/Lectures/ planargraphs.pdf, 2011.

[8] M. Ch. Golumbic and A. Mintz, Factoring Logic Functions Using Graph Partitioning, in: Proc. IEEE/ACM Int. Conf. Computer Aided Design, 1999, 109-114.

[9] M. Ch. Golumbic, A. Mintz, and U. Rotics, Factoring and Recognition of ReadOnce Functions using Cographs and Normality, in: Proc. 38th Design Automation Conf., June 2001, 195-198.

[10] M. Ch. Golumbic and Y. Perl, Generalized Fibonacci Maximum Path Graphs, Discrete Mathematics 28, 1979, 237-245. 
[11] A. Jain and N. Chandrasekharan, An Efficient Parallel Algorithm for Min-Cost Flow on Directed Series-Parallel Networks, in: Proceedings of the 7th International Parallel Processing Symposium, Newport Beach, California, USA, April 1993, 188192.

[12] M. Korenblit and V. E. Levit, On Algebraic Expressions of Series-Parallel and Fibonacci Graphs, in: Discrete Mathematics and Theoretical Computer Science, Proc. 4th Int. Conf., DMTCS 2003, LNCS 2731, Springer, 2003, 215-224.

[13] M. Korenblit and V. E. Levit, Square Rhomboids and Their Algebraic Expressions, in: Proc. 2009 Int. Conf. on Theoretical and Mathematical Foundations of Computer Science (TMFCS-09), 2009, 110-117.

[14] M. Korenblit and V. E. Levit, An Improved Full Decomposition Algorithm for Generating Algebraic Expressions of Square Rhomboids, in: Proc. 2010 Int. Conf. on Theoretical and Mathematical Foundations of Computer Science (TMFCS-10), 2010, 31-38.

[15] V. E. Levit and M. Korenblit, A Symbolic Approach to Scheduling of Robotic Lines, in: Intelligent Scheduling of Robots and Flexible Manufacturing Systems, The Center for Technological Education Holon, Israel, 1996, 113-125.

[16] C. L. Monma, Sequencing with Series-Parallel Precedence Constraints, Mathematics of Operations Research, Vol. 4, No 3, 1979, 215-224.

[17] C. L. Monma and J. B. Sidney, The Two-Machine Maximum Flow Time Problem with Series-Parallel Precedence Constraints: An Algorithm and Extensions, Operations Research, Vol. 27, No 4, 1979, 792-798.

[18] D. Mundici, Functions Computed by Monotone Boolean Formulas with no Repeated Variables, Theoretical Computer Science 66, 1989, 113-114.

[19] D. Mundici, Solution of Rota's Problem on the Order of Series-Parallel Networks, Advances in Applied Mathematics 12, 1991, 455-463.

[20] M. K. Oikawa, J. E. Ferreira, S. Malkowski, and C. Pu, Towards Algorithmic Generation of Business Processes: From Business Step Dependencies to Process Algebra Expressions, in: Business Process Management, Proc. 7th Int. Conf., BPM 2009, LNCS 5701, Springer, 2009, 80-96.

[21] T. Politof and A. Satyanarayana, Efficient Algorithms for Reliability Analysis of Planar Networks - A Survey, IEEE Transactions on Reliability, Vol. R-35, No 3, 1986, 252-259.

[22] J. S. Provan and R. C. Burk, Two-Connected Augmentation Problems in Planar Graphs, Journal of Algorithms 32, 1999, 87-107.

[23] Handbook of Discrete and Combinatorial Mathematics, edited by K. H. Rosen, CRC Press, Boca Raton, 2000.

[24] A. Satyanarayana and R. K. Wood, A Linear Time Algorithm for Computing KTerminal Reliability in Series-Parallel Networks, SIAM Journal of Computing, Vol. 14, No 4, 1985, 818-832. 
[25] P. Savicky and A. R. Woods, The Number of Boolean Functions Computed by Formulas of a Given Size, Random Structures and Algorithms 13, 1998, 349-382.

[26] A. Tamir, A Strongly Polynomial Algorithm for Minimum Convex Separable Quadratic Cost Flow Problems on Two-Terminal Series-Parallel Networks, Mathematical Programming 59, 1993, 117-132.

[27] J. A. Wald and C. J. Colbourn, Steiner Trees in Probabilistic Networks, Microelectronics and Reliability, Vol. 23, No 5, 1983, 837-840.

[28] A. R. R. Wang, Algorithms for Multilevel Logic Optimization, Ph.D. Thesis, University of California, Berkeley, 1989. 